\title{
Las Ciencias instrumentales en la Investigación Biomédica
}

\section{Instrumental Sciences in Biomedical Research}

Josep Roma Millán

Fundació Bosch Gimpera. Universitat de Barcelona. Barcelona

Hay una serie de ciencias que se hacen imprescindibles para poder investigar e interpretar los resultados científicos, son la ciencias que llamamos instrumentales 0 auxiliares. Entre ellas se encuentran la Demografía, la Epidemiología y la Bioestadística. Además, hay que tomar en consideración las técnicas de investigación cualitativa, el conjunto de estrategias e instrumentos de búsqueda de información bibliográfica y, también las metodologías de presentación de resultados. Finalmente, no puede olvidarse la ética, en sus dos componentes de bioética y de ética del trabajo científico, si queremos desarrollar un trabajo siguiendo el método científico.

Este capítulo explica cuál es la función de estas disciplinas en el seno de la investigación científica y del desarrollo de proyectos.

Palabras clave: Ciencias instrumentales, Demografía, Epidemiología, Bioestadística, Bioética, Investigación Cualitativa
Some scientific disciplines are essential for research and scientific results interpretation. Instrumental or auxiliary sciences include Demography, Epidemiology, and Biostatistics. Also, it is necessary to take into account the techniques for qualitative research, the strategies and instruments for bibliographic information and the methodology for scientific results presentation. Finally, to develop a project according to the scientific method, it is necessary to consider ethics, in its two components: bioethics and the ethics of scientific method.

This report explains which is the function of these instrumental and auxiliary sciences in the context of the scientific research and the development of scientific projects.

Key words: Instrumental Sciences, Demography, Epidemiology, Biostatistics, Bioethics, Qualitative Research

\section{INTRODUCCIÓN}

Para investigar es preciso partir de una buena pregunta. Tener la curiosidad, incluso la necesidad, de dar respuesta a algo sobre lo que no tenemos respuesta, es lo que da inicio a cualquier proyecto de investigación. Naturalmente que para contestar a la
Correspondencia:

Josep Roma Millán

Fundació Bosch Gimpera.

Universitat de Barcelona.

E-mail: jroma@fbg.ub.es pregunta será preciso bucear en el conocimiento de referencia y desarrollar métodos que son propios de dicho conocimiento. Los estabularios y laboratorios son buenos ejemplos de ello.

Pero muchas veces la investigación debe desarrollarse en seres humanos, en colectivos. En todos estos casos hay una serie de ciencias que podemos llamar instrumentales o auxiliares que se hacen imprescindibles para poder llevar a cabo la investigación e interpretar correctamente sus resultados.

En realidad se trata de aplicar lo que entendemos por método científico con el rigor necesario. Partimos de la necesidad de observar para plantear hipótesis de trabajo y seguimos con la necesidad de contrastar dicha hipótesis. Finalmente hará falta generalizar 
los resultados para someterlos constantemente a posibles falsaciones. Para desarrollar todos estos procesos adecuadamente se hace imprescindible contar con las anunciadas ciencias instrumentales.

El pensamiento lógico y la racionalidad matemática acostumbran a fundamentar estas disciplinas, de manera que en muchas ocasiones hace imprescindible el planteo de un trabajo interdisciplinario para poder llevar a buen puerto un proyecto. Ello lleva aparejado un debate de difícil solución para determinar hasta qué punto es necesario para un investigador el conocimiento de dichas materias. Encontrar el punto de equilibrio entre el conocimiento propio y la ayuda del especialista va a depender casi siempre del tipo de investigación, del ámbito concreto en que se practica, y también con frecuencia, del desarrollo de recursos humanos dedicados a la investigación del propio país.

Consideramos entre dichas ciencias a la Demografía, la Epidemiología, la Bioestadística, las técnicas de investigación llamadas cualitativas, el conjunto de estrategias e instrumentos de búsqueda de información bibliográfica y, también aunque no sea propiamente una ciencia, las metodologías de presentación de resultados. Finalmente creemos que la ética, en sus dos componentes de bioética y de ética del trabajo científico, han adquirido un rango de extraordinaria importancia sin cuyos aportes tampoco podemos concebir el buen desarrollo del método científico.

En este pequeño capítulo pretendemos explicar cuál es la función de estas distintas disciplinas en el seno de la investigación y del desarrollo de proyectos. Dejamos para módulos y enseñanzas más avanzados y de detalle, la posibilidad de aprender formalmente cada una de las disciplinas.

\section{DEMOGRAFÍA}

La demografía consiste básicamente en el estudio de las poblaciones. Dado que en muchas ocasiones la investigación se basa en individuos o poblaciones humanas, es necesario conocer elementos del comportamiento de las comunidades para dilucidar e interpretar los resultados de las investigaciones.

La descripción de una población incluye el conocimiento de fenómenos o realidades que luego van a resultar importantes para el análisis de variables relacionadas con el ámbito de la salud: natalidad, mortalidad, fecundidad, nupcialidad, migraciones.

La mortalidad es de por si un fenómeno que muchas veces describe el resultado de intervencio- nes sanitarias de todo tipo: fármacos de nueva implantación, cambios en los hábitos de vida, determinadas tendencias en alimentación, factores exógenos que intervienen en la génesis de enfermedades. Los otros fenómenos demográficos mencionados explican en buena medida la composición y hábitos de la población en distintos grupos de edades, además de resultar también muchas veces factores explicativos de enfermedades.

El conocimiento de la demografía implica así mismo el manejo de grandes bancos de datos poblacionales que son recurso habitual de información en diversos proyectos de investigación. Censos, padrones municipales, estadísticas vitales de mortalidad con todos sus detalles, registros e informes de morbilidad, son los más conocidos.

Los datos demográficos constituyen información básica en los denominadores de los principales indicadores y tasas que se utilizan en el ámbito biomédico. La construcción de tasas, ratios y proporciones es también tarea habitual en demografía. Dichos cálculos acaban siendo transcendentales para la elaboración de hipótesis estadísticas y para su contraste.

\section{EPIDEMIOLOGÍA}

La Epidemiología estudia la distribución de las enfermedades en la población y analiza sus causas y consecuencias. Necesita de la demografía para trabajar con los datos propios de las poblaciones de estudio, pero va más allá: analiza y sigue los procesos de enfermar, su génesis y su pronóstico.

De una parte describe aquello que ocurre en cuanto a enfermar se refiere. Fotografía pues la realidad de una comunidad: cuáles son las enfermedades que existen, en qué grupos de edad, con qué diferencia entre los géneros, si las frecuencias son distintas entre etnias u otros subgrupos de la población. Para ello utiliza indicadores que son propios de la descripción: tasas de mortalidad, prevalencias e incidencias de enfermedades.

De otra parte analiza la realidad. Trata de establecer relaciones de causa y efecto para así poder establecer políticas de prevención y promoción. Trata también de comprobar la eficacia de medicamentos y demás estrategias farmacológicas, para así establecer políticas de asignación de recursos en la asistencia sanitaria. Para ello utiliza indicadores o medidas de asociación: riesgo relativo y riesgo atribuible sobre todo.

En las últimas décadas la epidemiología utiliza 
también sus metodologías para analizar y conocer el funcionamiento de los servicios de salud. Para ello utiliza diversos indicadores de funcionamiento de dichos servicios (índices de ocupación, tasas de frecuentación, etc.), indicadores de resultados asistenciales (porcentaje de mejora o curación) e incluso económicos (medidas de coste-efectividad o de costeutilidad). En líneas generales hablamos de investigación de servicios de salud.

Finalmente la Epidemiología estudia como efectuar los contrastes de hipótesis. Es decir, cuál es la estrategia de confección de grupos testigo y grupos de intervención. Algo que resulta claro es que no se puede establecer un contraste con finalidades analíticas sin disponer de un grupo sobre el que se interviene y de otro grupo sobre el que no se interviene y actúa como testigo. Las diversas estrategias de diseño de estos grupos constituye un apartado de suma importancia por su complejidad posible.

Es preciso finalizar este capítulo resaltando la gran aportación de la informática y la estadística en el tratamiento y análisis de diversas variables al mismo tiempo. Lógicamente el desarrollo de las enfermedades no puede reducirse sólo a la relación entre dos variables. Ello ha constituido la base de la Epidemiología durante muchas décadas. Ahora podemos conocer como diversas variables influyen en la génesis de una enfermedad, o en su alivio o curación, y además podemos calcular el peso o importancia de cada una de ellas. La regresión logística y la regresión multivariada han emergido como los métodos de análisis más populares en los últimos tiempos.

\section{BIOESTADÍSTICA}

La bioestadística es una disciplina matemática que trata de analizar numéricamente los datos y variables relacionados con la enfermedad, su génesis y su pronóstico. La materia básica con la que trata son las variables y sus mediciones. La medición y sus métodos forma parte de los fundamentos de la disciplina. Medir correctamente es un aspecto imprescindible para poder desarrollarse en los ámbitos de la demografía y la epidemiología.

También aquí podemos hablar de estadística descriptiva para estudiar como presentar resultados de lo que ocurre. En realidad se trata de describir con el máximo detalle posible lo que ya hemos empezado a realizar en las anteriores disciplinas. Sólo así se puede llegar a entender con toda precisión el comportamiento numérico de una variable.
En cambio la estadística que llamamos inferencial cubre otro aspecto clave de la investigación. Se trata de poder realizar afirmaciones de lo que ocurriría en toda la población a partir de los resultados de una muestra de la misma. Dado que en general es imposible, por razones logísticas y económicas, trabajar con toda la población, nos vemos obligados a hacerlo sólo con una pequeña parte de ella, a la que llamamos muestra. El proceso de inferencia lleva consigo toda la complejidad de la abstracción matemática y es imprescindible en el proceso de contraste cuando actuamos con muestras.

Tal y como hemos mencionado anteriormente, la informática ha sido de gran ayuda a la bioestadística para el desarrollo de potentes métodos de análisis multivariante. Ello ha permitido un conocimiento más cercano de la realidad en toda su sofisticación.

\section{INVESTIGACIÓN CUALITATIVA}

Recientemente se han desarrollado una serie de instrumentos de evaluación que se agrupan bajo la etiqueta de investigación cualitativa. A veces se confunde con el análisis de variables cualitativas en estadística, pero no es el caso. Se trata en realidad de una metodología que no parte del concepto de muestra representativa aunque sí tiene como objetivo el llegar a realizar inferencia hacia la población.

El objetivo de la investigación cualitativa es llegar a la descripción y conocimiento de detalles sobre las distintas variables de estudio que un análisis estadístico y cuantitativo clásico no permite. Para ello se utilizan en general transcripciones del lenguaje verbal y escrito de forma completa y se analizan tratando de dar respuesta a lo que se busca. Con ello la posibilidad de mezclar argumentos y consideraciones subjetivas del investigador es grande, pero existen una serie de técnicas que pretenden evitarlo.

En muchas ocasiones la muestra no se selecciona al azar sino que se construye a partir de la descripción de los subgrupos que se pretende analizar y se busca aquellas personas más idóneas y que mejor información, o sea de más calidad, puedan aportar a la investigación.

En líneas generales podemos afirmar que la investigación cualitativa tiene gran valor en las fases prospectivas, de detección de necesidades y de aproximación primera a una pregunta de difícil respuesta. Su combinación con el análisis cuantitativo en una segunda fase confirmativa y de conclusiones generalizables puede ser de gran utilidad al planteamiento general de métodos a seguir en una inves- 
tigación. En realidad tiene grandes ventajas en la fase de observación detallada ante una buena pregunta y previamente a plantearse la forma concreta de contraste.

\section{INVESTIGACIÓN BIBLIOGRÁFICA}

En las últimas décadas la expansión y crecimiento del conocimiento ha alcanzado límites impensables cuando dicha disciplina llegó a ser fundamental en el siglo XIX. Las revistas científicas han sido, y de hecho siguen siendo, los instrumentos de difusión del conocimiento más eficaces y populares. Aquí también la informática ha permitido avances substanciales en el manejo y gestión de la misma de forma que ningún investigador en ningún campo puede permitirse el lujo de prescindir de ellos.

La información bibliográfica que aparece en revistas es sistemáticamente compilada y almacenada en bases de datos informatizados, de manera que su manejo se hace sencillo si se conocen estos medios. El Medline es la más popular de dichas bases de datos ya que agrupa la información de tipo biomédico y también de disciplinas que en realidad dependen del mismo (gestión en salud, salud pública, educación médica). Pero existen otras bases de datos importantes en los ámbitos de la Psicología, las Ciencias de la Educación, la propia Salud Pública, que también pueden ser útiles al investigador biomédico.

Con las posibilidades del manejo de la información que dan estos medios, el investigador puede estar permanentemente actualizado sobre los avances del conocimiento en su campo de estudio. Además hoy es posible hacerlo prácticamente sentado en un ordenador conectado a la red, sin necesidad de tener que acudir a las bibliotecas. Por este motivo, éstas se están convirtiendo en virtuales, espacios en la red que permiten al investigador entrar y manejarse para conocer y tener a su alcance toda la información disponible en la biblioteca.

\section{PRESENTACIÓN DE RESULTADOS}

Por la misma razón que antes aludíamos, la creciente expansión del conocimiento, su génesis a partir de centros y equipos cada vez más numerosos y situados en los cinco continentes del mundo, al investigador se le hace imprescindible saber como presentar los resultados de sus trabajos de forma comprensible y que finalmente resulte también al alcance de toda la comunidad científica.
En primer lugar está la presentación en comunicaciones orales, algo cada vez más extendido. El investigador debe ser capaz de resumir su trabajo en muy poco espacio de manera que resulte comprensible para los árbitros que le van a juzgar, pero también para los colegas que van a orientar sus intereses a partir de esta primera lectura. En segundo lugar debe ser capaz de preparar una presentación oral también limitada en el tiempo y combinar sus habilidades de presentar en público una idea con el soporte gráfico necesario para que resulte perfectamente comprensible.

En segundo lugar está la presentación escrita en artículos a revistas. Sabemos que éste es el objetivo final de cualquier trabajo de investigación puesto que es realmente el que acaba validando el mismo ante la comunidad y el que sirve para mejorar su posición. Ahí también se parte de un primer resumen escrito, pero después deben combinarse buenas técnicas de redacción y de síntesis con las representaciones gráficas más adecuadas de apoyo al texto escrito. Existen reglas generales que se deben seguir, y por tanto aprender, y existen después reglas propias de cada revista que el investigador debe aprender a seguir y adecuar.

Todavía habría un tercer aspecto que se hace cada día más importante. Se trata de comunicar los resultados y avances del conocimiento al gran público. Hay un primer interés más relacionado con lo que entendemos como aumento de cultura y de capacidad de entender el mundo, pero hay también un interés digamos que egoísta, en el buen sentido del término, de saber qué debe hacer el ciudadano como paciente o como familiar de un enfermo. Los instrumentos propios de internet han puesto al alcance de todo el mundo la información médica. Por tanto, lo que era un deber moral constituye actualmente una necesidad profesional, ya que el diálogo con los pacientes se fundamente cada vez más sobre la base de un intercambio de información. El profesional podrá guiar dicho proceso si previamente es capaz de explicar correctamente el conocimiento que se genera.

\section{ÉTICA EN LA INVESTIGACIÓN}

Los planteamientos éticos están siendo cada día más necesarios para el investigador. Ello está relacionado con aspectos que citábamos en el último apartado: la comunidad es cada vez más consciente de las posibilidades del conocimiento para mejorar su salud y su calidad de vida, de modo que intervie- 
ne más en la orientación de su génesis y en la gestión de sus procedimientos. A partir de ahí es lógico que se planteen dilemas morales y por tanto que existan posicionamientos éticos de los grupos sociales y comunidades en función de sus objetivos e intereses.

Debemos separar de entrada lo que serían los problemas y dilemas propios de la bioética, es decir aquellos que se derivan del uso de los propios avances de las ciencias biológicas, de los dilemas estrictamente profesionales que se derivan de las normas y quehaceres propios de los científicos.

En el primer caso nos encontramos con todos los dilemas surgidos de los límites de la vida y sus usos. Son dilemas que se comparten lógicamente con la comunidad médica y asistencial en general. El uso de óvulos congelados afecta a los investigadores que trabajan en este ámbito, pero también a médicos, enfermeras y biólogos que tratan de inducir embarazos en aquellos pacientes que les expresan dicha necesidad. Por lo tanto los dilemas bioéticos se comparten con toda la comunidad profesional.

La ética de la profesión investigadora afecta a las normas propias de su tarea. Cómo trabajar con pacientes en los grupos de investigación y obtener consentimiento informado, cómo seguir las reglas propias de la investigación sin caer en la tentación de ahorrar pasos que permitan concluir "aquello que se desea", cómo comunicar correctamente y con sinceridad los resultados, son ejemplos de la importancia de la reflexión ética en el trabajo científico.

\section{Sesión de trabajo \\ Prof. C. González, Profa. N. García-López y Prof. A. Agustí}

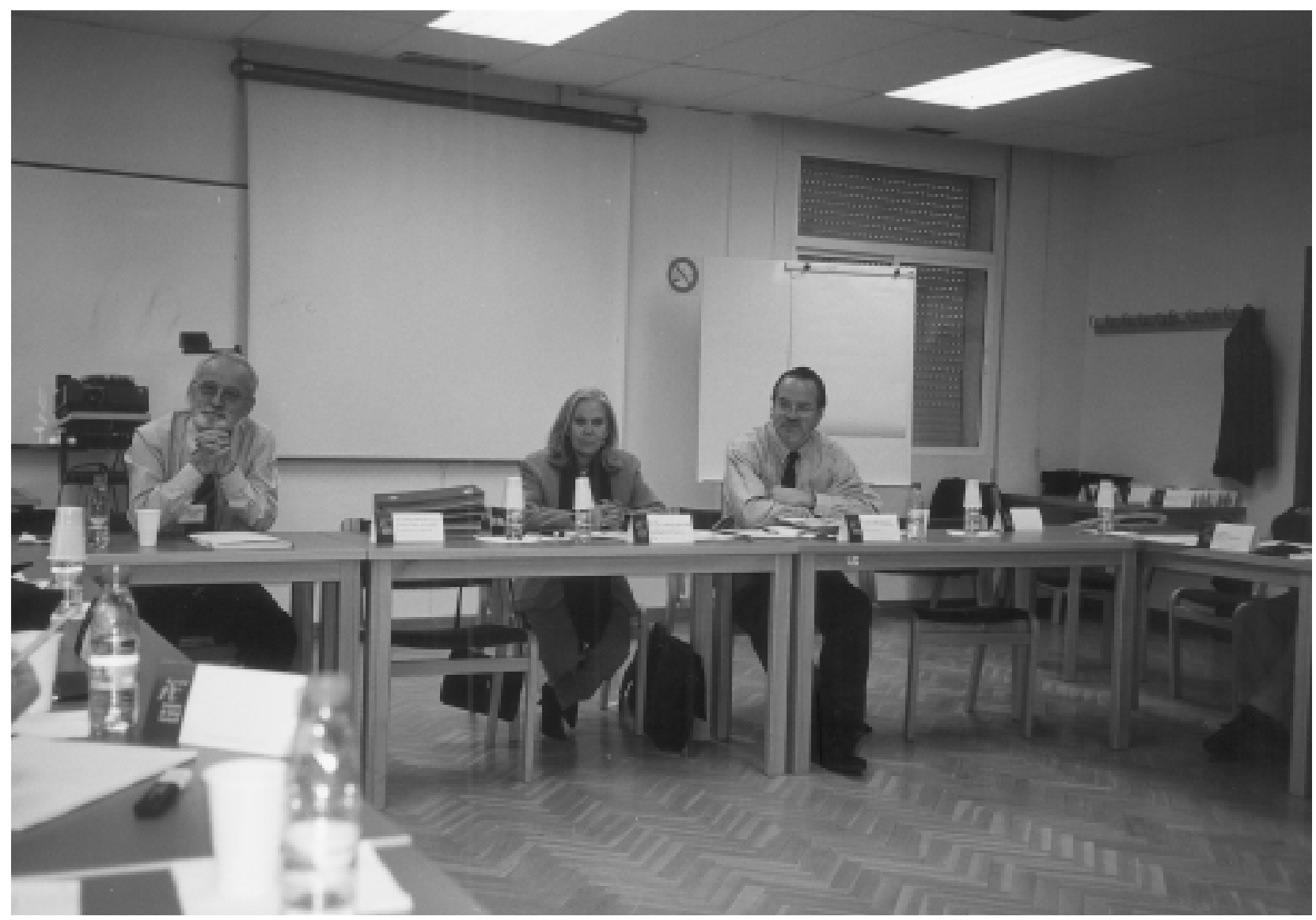

